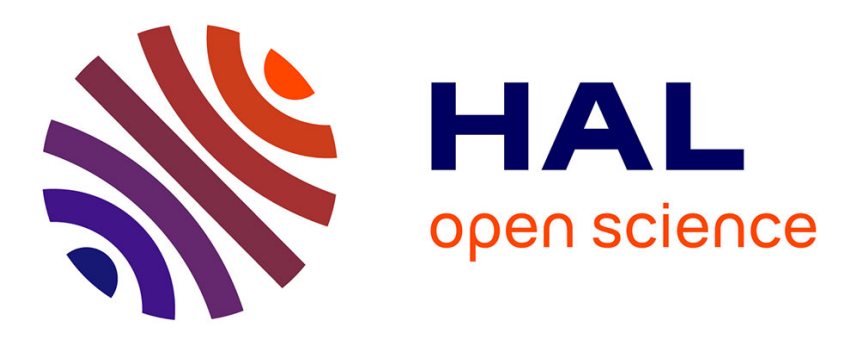

\title{
Head and trunk mass and center of mass position estimations in able-bodied and scoliotic girls
}

Mohsen Damavandi, Georges Dalleau, Georgios A. Stylianides, Charles-Hilaire Rivard, Paul Allard

\section{- To cite this version:}

Mohsen Damavandi, Georges Dalleau, Georgios A. Stylianides, Charles-Hilaire Rivard, Paul Allard. Head and trunk mass and center of mass position estimations in able-bodied and scoliotic girls. Medical Engineering \& Physics, 2013, 35 (11), pp.1607-1612. 10.1016/j.medengphy.2013.05.010 . hal01232308

\section{HAL Id: hal-01232308 \\ https: / hal.univ-reunion.fr/hal-01232308}

Submitted on 27 Oct 2016

HAL is a multi-disciplinary open access archive for the deposit and dissemination of scientific research documents, whether they are published or not. The documents may come from teaching and research institutions in France or abroad, or from public or private research centers.
L'archive ouverte pluridisciplinaire HAL, est destinée au dépôt et à la diffusion de documents scientifiques de niveau recherche, publiés ou non, émanant des établissements d'enseignement et de recherche français ou étrangers, des laboratoires publics ou privés. 


\title{
Head and trunk mass and center of mass position estimations in able-bodied and scoliotic girls
}

\author{
Mohsen Damavandi ${ }^{\mathrm{a}, \mathrm{b}, *}$, Georges Dalleau $^{\mathrm{b}, \mathrm{c}}$, Georgios Stylianides ${ }^{\mathrm{d}}$, Charles-Hilaire \\ Rivard $^{\mathrm{e}, \mathrm{f}}$, Paul Allard ${ }^{\mathrm{b}, \mathrm{e}}$ \\ a Faculty of Physical Education and Sport Sciences, Hakim Sabzevari University, Sabzevar, Iran \\ ${ }^{\mathrm{b}}$ Department of Kinesiology, University of Montreal, Montreal, QC, Canada ${ }^{1}$ \\ ' CURAPS-DIMPS, Faculté des Sciences de l'Homme et de l'Environnement, Université de la Réunion, Le Tampon, France \\ d Department of Exercise Science and Sport, University of Scranton, PA, USA \\ e Human Movement Laboratory, Research Center, Saint-Justine Hospital, 3175 Côte Sainte-Catherine, Montreal, QC, Canada H3T 1C5 \\ ${ }^{\mathrm{f}}$ Department of Orthopedic Surgery, Sainte-Justine Hospital, Montreal, QC, Canada
}

\begin{abstract}
A B S T R A C T
Anthropometric tables are not applicable to calculate the scoliotic trunk mass and center of mass (COM). The purposes of this study were: (1) to estimate the head and trunk mass and COM in able-bodied and scoliotic girls using a force plate method, (2) to estimate head and trunk COM offset compared to those of the body, and (3) the use of mean ratios to estimate the head and trunk COM calculated in this study and that calculated according to a conventional three-dimensional (3D) method compared to the measured values. Twenty-one scoliotic and twenty able-bodied girls participated. The subjects stood upright with arms beside the trunk on a force plate that collected data at $60 \mathrm{~Hz}$ for a period of $5 \mathrm{~s}$. The anteroposterior and mediolateral positions of the body COM were obtained from the mean center of pressure values. The height of the body COM was estimated by the reaction board method. Afterwards a body segment was displaced and changes in force plate readings were recorded and applied to estimate the head and trunk mass and COM. Trunk offset was defined as the difference between the COM of the body and head and trunk. The measured head and trunk COM was compared to values obtained by the mean ratios calculated from this study and given by the conventional 3D method. The relative head and trunk mass and the anteroposterior trunk offset were larger in scoliotic girls. The force plate method gave similar results to measured COM values for both groups underlying its capability to provide a more accurate estimation of COM related values. Thus, the use of mean ratios of 0.5538 and 0.6438 obtained in this study to estimate the head and trunk mass and COM position in scoliotic girls can overcome the main drawbacks of current anthropometric methods, if direct measurements cannot be taken.
\end{abstract}

\section{Introduction}

Body segment masses and center of mass (COM) positions are required to estimate joint reaction forces and muscle moments during gait or other physical activities. These values are often calculated from anthropometric tables assembled from cadaver data comprising of a limited number of elderly male Caucasians [1,2]. Though they are well suited for populations similar to those from

\footnotetext{
* Corresponding author at: Faculty of Physical Education and Sport Sciences, Hakim Sabzevari University, Sabzevar, Iran. Tel.: +98 5714004411.

E-mail addresses: mohsen.damavandi1@gmail.com,

mohsen.damavandi@hotmail.com (M. Damavandi).

1 The work should be attributed to these departments.
}

which the anthropometric measures were obtained, their applications are questionable for children [3], adolescents [4], obese individuals [5], and scoliotic patients [6].

Imaging techniques using magnetic resonance imaging (MRI) [5], dual energy X-ray absorptiometry [7], and gamma-ray scanning [8] were developed as alternative approaches to determine body segment parameters (BSP) based on individuals outside the Caucasian male population and on living subjects. Though appealing, the findings of these techniques can still not be applied to individuals outside the subjects tested due to wide range of differences in BSP between various populations [7]. Imaging techniques are also limited due to radiation exposure, time, expense, and logistical issues [5]. Furthermore, Damavandi et al. [9] questioned their use in lean and obese able-bodied adults. Even so, Engsberg et al. [10] were the first to report the whole body COM position in three 
scoliotic adults using MRI. Nonetheless, little is known about the mass and COM of the trunk in scoliotic girls and how they compare to non-scoliotic girls.

Damavandi et al. [9] proposed a new force plate method to determine subject-specific segment masses and COM positions. It was tested in 23 able-bodied young adults with body mass index (BMI) ranging from 17.6 to $31.9 \mathrm{~kg} / \mathrm{m}^{2}$ to verify its sensitivity. Though results were within the range of those of the other literature-based methods [11-13] for all subjects tested, statistical differences were identified between the lean and obese subjects in the relative head and trunk COM position. This personalized method could be applied to estimate the mass and COM of body segments in scoliotic girls and particularly the head and trunk segment where the threedimensional (3D) spinal deformation is responsible for geometric changes in the trunk and rib cage $[14,15]$.

Knowing the relative position of the COM of trunk with respect to that of the whole body could be important to spine surgeons since it complements sagittal balance. Sagittal balance describes the position of the head relative to the pelvis in the sagittal plane reflecting the postural attitude assumed by the patient [16]. Considering that the head and trunk segment represents about $60 \%$ of the body weight, repositioning the COM of the scoliotic trunk in relation to the line of gravity passing through the body COM could improve the stability of the correction with a body brace or spinal instrumentation and reduce back pain through reducing muscle forces and moments applied on the spine. In addition to surgeons, more accurate estimations of the trunk mass and COM position are of interests of biomechanists to analyze energy cost of waking in scoliotic patients [17] and evaluate loads on the lumbar spine [18], as well as ergonomists in scrutinizing lifting mechanics [19]. To our knowledge no one has yet determine the COM of trunk in either able-bodied or scoliotic girls.

The objectives of this study are to estimate the head and trunk mass and COM position using the personalized force plate method described by Damavandi et al. [9] in able-bodied and scoliotic girls since girls are more at risk of developing a spinal deformity [20]. The head and trunk COM positions are afterwards compared to those of the whole body to estimate COM offset along the anteroposterior, mediolateral, and vertical axes in both groups. Since ratios are often used we wish to determine if those calculated for able-bodied and scoliotic girls in this study and the one given by a well-accepted and conventional 3D method [11] can be used to approximate the measured the head and trunk COM positions. Our hypothesis is that current anthropometric ratios do not accurately estimate the mass and the COM position of the scoliotic head and trunk segment.

\section{Method}

Twenty-one scoliotic girls who participated to this study diagnosed as adolescent idiopathic scoliosis by an orthopedic surgeon according to the criteria defined by Bunnel [21] and from a frontal plane radiograph. All subjects had a right thoracic curve of about $13^{\circ}$ (range $5-28^{\circ}$ ) as reported in Table 1 and none was under active treatment. A group of 20 able-bodied girls formed the control group. They were examined by the orthopedic surgeon to ensure all were in general good health. The two groups were comparable in age $(p=0.300)$, height $(p=0.068)$, weight $(p=0.168)$ and BMI $(p=0.485)$ as detailed in Table 1 . Both girls and parents signed the informed consent form approved by the Hospital Ethics Committee.

The procedure to estimate the head and trunk mass as well as its COM position was based on the work of Damavandi et al. [9]. Though the reader is referred to this work for further information the method is briefly described here. It lies on changes in force plate readings taken in two different positions. The subject stood upright on the force plate (AMTI, Model OR6-5, Newton, MA, USA) with arms beside the trunk. Afterward data were collected at $60 \mathrm{~Hz}$ for a period of $5 \mathrm{~s}$ and then filtered by a fourth-order low-pass Butterworth filter with a $6 \mathrm{~Hz}$ cutoff frequency. The anteroposterior (AP) and mediolateral (ML) positions of the whole body COM were obtained from the mean center of pressure (COP) [22]. The height of the COM was estimated by the reaction board method described below. Then the whole body COM was calculated with respect to the mid-hip position.

Afterwards a body segment was displaced; for example, the right upper limb was flexed at $90^{\circ}$ and the mean COP was determined once more. Since the whole body less the displaced segment COM remains the same for the initial (i.e. with arms alongside the trunk) and final (i.e. $90^{\circ}$ flexion of right upper limb) positions, the mass of the displaced segment can be calculated. Since there was a difference of less than $2 \%$ upper limb's mass and COM position values between the method proposed by Damavandi et al. [9] and that of de Leva [11] the inclusion of shoulder in the trunk mass can be considered negligible. The upper and lower limb masses were calculated using the same procedure. Then, the head and trunk mass was estimated as the difference between the whole body mass and that of the upper and lower limbs. This value was normalized with respect to that of the whole body.

The COM position of the body segments was estimated using the reaction board method where the subject lies on a heavy and rigid board supported at one end by the force plate and a triangular support at the other end. Again the subject was required to assume two postural configurations while lying on the board. Force plate data were first obtained with the arms alongside the trunk. This was followed by a body segment displacement (e.g. $90^{\circ}$ right upper limb flexion). This procedure was performed for the other upper and lower limbs and for all subjects. Afterwards, head and trunk COM location was also calculated with respect to the midhip position. The COM offset was defined as the difference between the whole body COM position and that of the head and trunk along each axis for each subject. Lying on a board could cause the deformity to assume a non-representative posture. Wessberg et al. [23] reported that the mean Cobb angle of axially loaded spines in MRI scans compared to a non-loaded ones were smaller by $8^{\circ}$ in idiopathic scoliosis patients. Even though the effect of gravity is taken in account it is still unknown if the loading device repositions the spine in the same 3D space.

Trunk and pelvis inclinations were also measured using a videobased system (Motion Analysis Corporation, Santa Rosa, USA). Six cameras were located around and at a distance of about $3 \mathrm{~m}$ from the subject standing upright and immobile on the force plate. Seven markers ( $16 \mathrm{~mm}$ diameter) were put over C7 (seventh cervical vertebra), greater trochanters, anterior superior iliac spines (ASIS), and posterior superior iliac spines (PSIS), as illustrated in Fig. 1. Video data were collected for $20 \mathrm{~s}$ at $60 \mathrm{~Hz}$ and filtered with a fourth-order zero-phase lag Butterworth filter having a cutoff frequency of $6 \mathrm{~Hz}$. Trunk inclination was calculated in the sagittal and frontal planes as the angle sustained by a line joining $\mathrm{C} 7$ and the mid-hip position. Pelvic tilt was calculated using the angle between the horizontal and a line connecting the ASIS and the PSIS [24].

The position of the head and trunk COM was reported as a fraction of the C7-mid-hip length and a mean value was calculated for both scoliotic and able-bodied girls. Then these mean values were used to recalculate the 3D coordinates of the head and trunk COM with respect to the mid-hip position and compared to the 3D measured coordinates and to values obtained by using a 0.6896 ratio given by de Leva [11] for the same body segment. Not only this is a conventional and well-accepted 3D method but also Damavandi et al. [9] found that it performed better than photographic and geometric approaches $[12,13]$ when tested in subjects of lean, normal and obese morphologies. 
Table 1

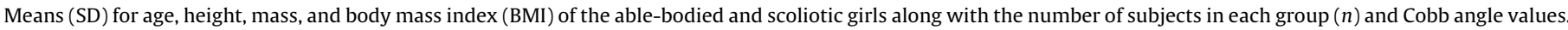

\begin{tabular}{|c|c|c|c|c|c|}
\hline Group & Age (yr) & Height $(\mathrm{cm})$ & Mass $(\mathrm{kg})$ & $\mathrm{BMI}\left(\mathrm{kg} / \mathrm{m}^{2}\right)$ & Cobb angle $\left(^{\circ}\right)$ \\
\hline Able-bodied $(n=20)$ & $12.5(1.3)$ & $156.3(7.8)$ & $43.7(6.9)$ & $17.9(2.4)$ & - \\
\hline Scoliotic $(n=21)$ & $11.7(3.1)$ & $148.4(17.0)$ & $40.0(13.3)$ & $18.2(3.2)$ & $13.5(5.5)$ \\
\hline
\end{tabular}

No significant difference was observed for age, height, mass and BMI ( $p \geq 0.068$ ).

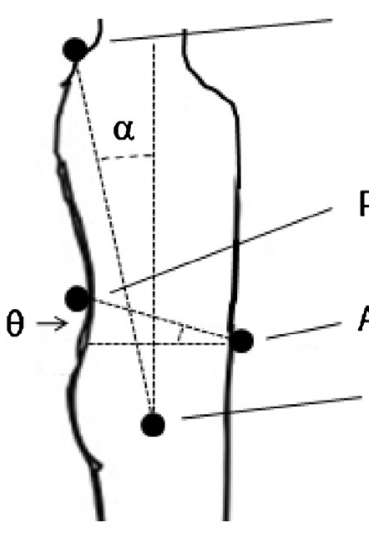

Sagittal view
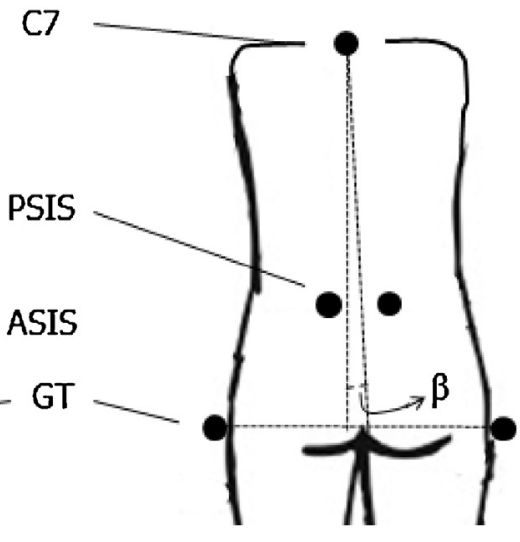

Frontal view
Fig. 1. Sagittal and frontal view illustrating anatomical landmarks used to measure trunk and pelvis inclinations. C7 = seventh cervical vertebra, PSIS $=$ posterior superior iliac spines, ASIS = anterior superior iliac spines, GT = greater trochanters, $\alpha=$ trunk inclination in the sagittal plane, $\beta=$ trunk inclination in the frontal plane, $\theta=$ pelvic tilt in the sagittal plane.

ANOVAs were performed on the head and trunk segment mass and COM as well as their normalized values with respect to the whole body mass and trunk length, respectively. ANCOVA were used with age, body mass, height and BMI as covariables for the sagittal and frontal trunk angles and pelvic tilt, head and trunk and whole body COM positions as well as their differences along each axis. To determine if there is a relation between the head and trunk COM position to the mid-hip position and the trunk length, Pearson coefficients of correlation were performed for both groups and $R^{2}$ values were calculated. Finally, repeated measures ANOVA were carried out on the head and trunk COM positions between the measured values and those calculated using the constant ratios given in this study and the one given by de Leva [11]. Post hoc analysis using a Bonferroni correction was carried out if a statistical difference was observed $(\alpha=0.05)$.

\section{Results}

For similar trunk lengths of approximately $525 \mathrm{~mm}$, the sagittal and frontal trunk inclination angles (both $p<0.001$ ) and pelvic tilt $(p=0.001)$ were different between able-bodied and scoliotic girls as presented in Table 2 . This reflects that the trunk inclination of the scoliotic girls was more toward the back by $3.8^{\circ}$, more inclined

Table 2

Means (SD) of head and trunk length, mass, and relative mass as well as trunk inclinations and pelvis tilt angles.

\begin{tabular}{llc}
\hline & $\begin{array}{l}\text { Able-bodied } \\
\text { subjects }\end{array}$ & Scoliotic subjects \\
\hline Head and trunk length $(\mathrm{mm})$ & $525.1(43.4)$ & $524.7(107.0)$ \\
Head and trunk mass $(\mathrm{kg})$ & $23.4(3.7)$ & $22.1(7.3)$ \\
Head and trunk mass/body mass & $0.5360(0.0089)$ & $0.5538(0.0167)^{\mathrm{a}}$ \\
Sagittal trunk flexion $\left(^{\circ}\right)$ & $9.7(2.4)$ & $5.9(1.6)^{\mathrm{a}}$ \\
Frontal trunk flexion $\left(^{\circ}\right)$ & $-0.6(1.3)$ & $1.8(1.0)^{\mathrm{a}}$ \\
Pelvis forward tilt $\left(^{\circ}\right)$ & $6.3(8.8)$ & $13.3(3.6)^{\mathrm{a}}$ \\
\hline
\end{tabular}

a Significant differences between the able-bodied and scoliotic subjects $(p<0.05)$. to the right by $2.4^{\circ}$, and with a $7.0^{\circ}$ greater forward pelvic tilt. Though the head and trunk mass was smaller by $1.3 \mathrm{~kg}$ in the scoliotic group its relative mass to the whole body was $3.3 \%$ greater at $0.5538(p<0.001)$.

The head and trunk as well as the whole body COM positions with respect to the mid-hip center are given in Table 3. The scoliotic group had their head and trunk segment COM more to the right by $15.5 \mathrm{~mm}(p<0.001)$, more posteriorly by $19 \mathrm{~mm}(p<0.001)$ and at about the same height $(p=0.316)$ as the able-bodied group. The relative position of the scoliotic head and trunk segment COM was about $4.1 \%$ lower at 0.6438 ( $p=0.017$ ) of the trunk's length than that of the able-bodied group. For the scoliotic girls their whole body COM was nearly $30 \mathrm{~mm}(p<0.001)$ more to the right and more anterior by nearly $9 \mathrm{~mm}(p=0.035)$ compared to the able-bodied girls.

Table 3 also provides the head and trunk offset for the three axes in both groups. The AP offset was larger in scoliotic girls with the whole body COM anterior to that of the head and trunk segment and the opposite occurred in able-bodied subjects (between groups, $p<0.001)$. In the ML direction this offset was negligible in the scoliotic girls compared to the able-bodied girls where the difference between groups was $14.9 \mathrm{~mm}(p<0.001)$.

Fig. 2 illustrates the relation between the head and trunk COM position to the mid-hip position and the trunk length for both groups. The $R^{2}$ values for the correlations are 0.8668 and 0.8721 for the able-bodied and scoliotic girls, respectively. Using three constant ratios namely, 0.6716 for able-bodied and 0.6438 for the scoliotic girls from the force plate method [9] as well as 0.6896 from de Leva [11], the 3D positions of the head and trunk segment COM are shown in Table 4. For the able-bodied girls there were statistical differences between the measured values and those of the de Leva [11] along the AP $(p=0.007)$ and vertical axes $(p=0.005)$. The values obtained from the de Leva [11] and the ratio of the proposed method were also significantly different in AP and vertical axes (both $p=0.000$ ). The largest mean error was $1 \mathrm{~mm}$ along the $\mathrm{AP}$ and ML axes and about $9 \mathrm{~mm}$ along the vertical axis for both methods.

For the scoliotic group, there was no statistical difference between the measured values and the proposed ratio of 0.6438 where $p$ values were higher than 0.367 . The values calculated by

Table 3

Means (SD) of head and trunk and whole body COM positions ( $\mathrm{mm}$ ) with respect to mid-hip position and their differences (offset) along anteroposterior (AP), mediolateral (ML), and vertical axes.

\begin{tabular}{lcc}
\hline & $\begin{array}{l}\text { Able-bodied } \\
\text { subjects }\end{array}$ & Scoliotic subjects \\
\hline Head and trunk COM (+forward) & $55.6(10.0)$ & $36.6(10.1)^{\mathrm{a}}$ \\
Head and trunk COM (+right) & $-3.6(7.9)$ & $11.9(6.1)^{\mathrm{a}}$ \\
Head and trunk COM (+up) & $352.7(31.6)$ & $336.3(65.0)$ \\
Head and trunk COM/trunk & $0.6716(0.0215)$ & $0.6438(0.0464)^{\mathrm{a}}$ \\
$\quad$ length & $44.6(12.4)$ & $53.7(6.6)^{\mathrm{a}}$ \\
Body COM (+forward) & $-17.7(9.4)$ & $12.7(9.4)^{\mathrm{a}}$ \\
Body COM (+right) & $51.7(23.2)$ & $63.3(34.2)$ \\
Body COM (+up) & $-11.0(15.9)$ & $17.1(10.6)^{\mathrm{a}}$ \\
AP offset & $-14.1(13.4)$ & $0.8(9.3)^{\mathrm{a}}$ \\
ML offset & $-300.9(28.4)$ & $-273.0(63.8)$ \\
Vertical offset &
\end{tabular}

a Significant differences between the able-bodied and scoliotic subjects $(p<0.05)$. 
Table 4

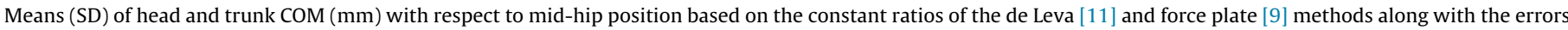
compared to the measured values for anteroposterior (AP), mediolateral (ML), and vertical axes.

\begin{tabular}{|c|c|c|c|c|c|}
\hline Method & Axis & Able-bodied subjects & Error & Scoliotic subjects & Error \\
\hline \multirow[t]{3}{*}{ de Leva [11] } & $\mathrm{AP}$ (+forward) & $56.6(10.4)^{\mathrm{a}}$ & 1.0 & $38.4(11.2)^{\mathrm{a}}$ & 1.8 \\
\hline & ML (+right) & $-3.6(8.1)$ & 0.0 & $12.4(6.4)^{\mathrm{a}}$ & 0.5 \\
\hline & Vertical (+up) & $362.1(29.9)^{a}$ & 9.4 & $361.8(73.8)^{a}$ & 25.5 \\
\hline \multirow[t]{3}{*}{ Force plate [9] } & $\mathrm{AP}$ (+forward) & $55.6(10.2)^{\mathrm{b}}$ & 0.0 & $36.7(10.8)^{b}$ & 0.1 \\
\hline & ML (+right) & $-3.6(7.9)$ & 0.0 & $11.8(6.1)^{\mathrm{b}}$ & -0.1 \\
\hline & Vertical (+up) & $352.6(29.1)^{\mathrm{b}}$ & -0.1 & $337.8(68.9)^{\mathrm{b}}$ & 1.5 \\
\hline
\end{tabular}

a Significant differences between the estimated and measured values $(p<0.05)$.

b Significant differences between the estimated values obtained from the constant ratios of the de Leva [11] and force plate [9] methods ( $p<0.05$ ).

the de Leva [11] method were statistically different from the measured and the proposed ratio values along the three axes $(p \leq 0.016)$. The largest mean error was an overestimation of the height of the head and trunk COM by about $25 \mathrm{~mm}$ with the de Leva [11] method though the errors along the other axes were less than $2 \mathrm{~mm}$.

\section{Discussion}

The first objective was to estimate the head and trunk mass and COM position using the personalized in vivo force plate method described by Damavandi et al. [9] in able-bodied and scoliotic girls. The head and trunk mass and whole body were identical in both groups. But, the ratio of head and trunk mass to that of the whole body of the scoliotic girls was higher (Table 2). This is explained by a slightly larger head and trunk mass to a lighter body mass for the scoliotic girls. This could be of clinical importance when loads are estimated on the spine since head and trunk mass is usually calculated from that of the whole body mass and anthropometric tables. Larivière and Gagnon [25] reported incertitude of up to 9\%
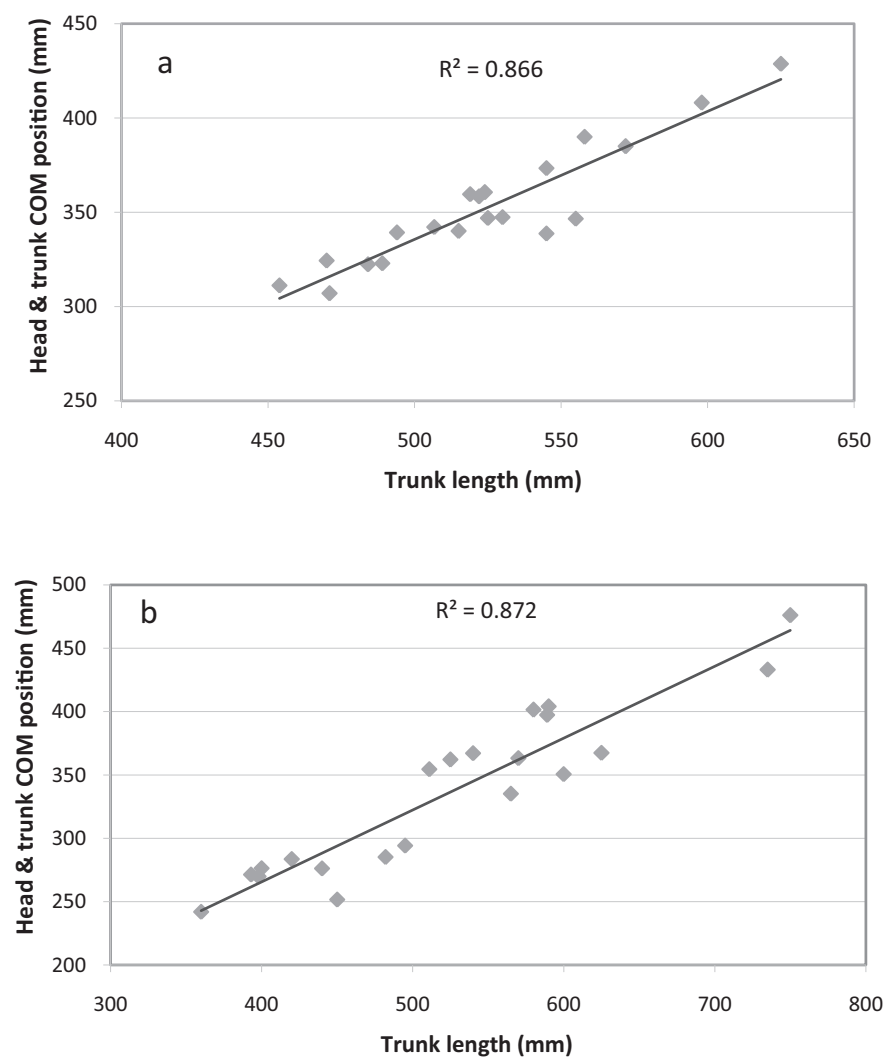

Fig. 2. Relation between the head and trunk center of mass (COM) position to the mid-hip position and the trunk length for (a) able-bodied and (b) scoliotic subjects. in the lumbar compression force according to the trunk inertial parameters used to estimate the lumbo-sacral moment. Using the ratio for women given by de Leva [11], the head and trunk mass of scoliotic girls was underestimated by $11.1 \%$. According to de Leva [11], head and trunk mass/body mass ratio is 0.4925 , while based on the force plate method [9] this ratio was 0.5538 (Table 2).

The main differences in the head and trunk COM position were observed in the horizontal plane where the scoliotic group was more to the right and closer to the mid hip position than that of the able-bodied girls (Table 3 ). Though a COM offset was expected in scoliotic girls it could appear unusual in able-bodied subjects. This is due to the whole body COM which is $17.7 \mathrm{~mm}$ to the left of the mid-hip position. It reflects a postural attitude where more weight is placed on a limb while standing upright $[26,27]$. This was observed by Dickstein et al. [28] who reported a 6\% difference in weight-bearing forces under the feet in standing. The whole body COM of scoliotic girls was more anterior and to the right than that of able-bodied girls. Though similar findings were reported by Fegoun et al. [29] and Engsberg et al. [10] these were for adult scoliotic subjects also lying in the supine position. To our knowledge these are the first values reported for both head and trunk segment and whole body COM in a population of young girls with and without a scoliosis.

The second objective was to assess trunk COM offset. This information could be important to the practicing surgeon when restoring sagittal balance [30]. According to Glassman et al. [31] restoration of sagittal balance should be a primary goal of spinal deformity surgery. In addition to postural alignment the surgeon could consider reducing the horizontal lever arm of the weight of the trunk and head to the line of gravity of the body or COM offset. This would reduce the torque on the spine and lessen back pain [32].

The scoliotic head and trunk COM position relative to their whole body COM (Table 3) can be explained by their greater forward pelvic tilt and a more backward trunk inclination (Table 2). Jackson et al. [33] and Nault et al. [34] observed a greater backward trunk inclination in their scoliotic group compare to their ablebodied girls, while Allard et al. [6] reported a hypokyphotic posture in ectomorphic scoliotic girls. It appears that in non-treated scoliotic girls where the Cobb angle is less than $28^{\circ}$, the COM position of the head and trunk is related to the trunk backward inclination.

The final objective was to test the use of a ratio to estimate the head and trunk COM position. Ratios have been commonly applied to estimate segmental COM position in healthy children [3], adults [11], and adolescents [4] but were not applied in scoliotic subjects with the exception of comparing methods [10]. For both able-bodied and scoliotic girls the $R^{2}$ values between the head and trunk COM position to the mid-hip position, calculated using ratios of the force plate method [9], and the trunk length were high justifying the use of a separate ratio to estimate the head and trunk COM position (Fig. 2). These ratios for able-bodied and scoliotic girls were 0.6716 and 0.6438 , respectively. The maximum difference in the 
head and trunk COM position was about $9 \mathrm{~mm}$ for the able-bodied girls regardless of the ratio used. This relatively low difference value can be explained by a small but statistically significant difference of about $2.7 \%$ between the ratio given by de Leva [11]; i.e. 0.6896 , and the ones given in this study for able-bodied girls; i.e. 0.6716.

For scoliotic subjects, the greatest difference between the estimated head and trunk COM position and the measured values was observed along the vertical axis when applying the ratio given by de Leva [11]. This corresponds to a 7.6\% difference which is similar to those calculated for the other directions (5.0\% for AP and $3.6 \%$ for $\mathrm{ML}$ ) using the same method. Using the proposed ratio (i.e. 0.6438 ) the relative difference is less than $1 \%$. The larger head and trunk mass relative to body mass of the scoliotic subjects (Table 2) explains in part the lower COM position of that segment compared to that of the able bodied-girls in this study (Table 3 ) as well as that of the ratio given by de Leva [11]. The height of the COM of the head and trunk segment was statistically lower with the proposed ratio compared to the de Leva [11] method. Similar finding was also reported by Engsberg et al. [10] in a comparison between subjectspecific (MRI) and literature-based [1] methods for the COM of the whole body.

The head and trunk COM position could be important when estimating the loads on the lumbar spine. Gum et al. [35] reported that the direction of transverse pelvic rotation varied according to the type of thoracic curve reflecting changes in the trunk COM location. Furthermore, changes in the COM location could affect the moment of inertia, according to the principal axis theorem, which would lead to a larger change in the kinetic output. During gait Mahaudens et al. [36] reported reduced pelvic, hip and shoulder motion amplitudes. This was explained by the stiffness of the deformity or by the bilateral prolonged activation timing of the lumbar and pelvic muscles. Since force plate data reflects the whole body COM motion [37], these observations could also be explained by the head and trunk COM position relative to the pelvis and by the shift in the position of the body COM as reported in this study and in Engsberg et al. [10].

Results from this study could be used as a guide to the surgeon in correcting scoliosis and other spinal deformities. They could be used in addition to the sagittal balance in the planning of the surgical treatment of adolescent idiopathic scoliosis. Knowing the COM offset or the distance between the trunk center of mass and that of the whole body to be reduced could be valuable in estimating the sagittal spinal curves that need to be restored by the instrumentation. This is particularly important since the trunk represents about $60 \%$ of the body mass. The COM offset provides an indication to the spine surgeon of the proximal vertebra and mostly that of the distal vertebra to instrument as well as the extent of sagittal curve to give to the rods. Similarly, the COM offset can be verified after fitting a body brace to ensure body and head and trunk alignment. Reducing the COM offset in scoliotic girls could diminish the perturbed horizontal control of the trunk during standing balance [38]. Furthermore, results of this study could be applied in order to more accurately assess energy expenditure during gait and estimate loads on the lumbar spine during various lifting techniques in able-bodied and scoliotic girls in clinical settings. The method proposed by Damavandi et al. [9] can be applied without a series of assumptions on body segment mass and COM position or using costly and time consuming imaging devices $[10,26]$.

\section{Conclusion}

In this study involving able-bodied and scoliotic girls the personalized force plate method described by Damavandi et al. [9] was applied to estimate the mass and COM position with respect to the mid-hip position. It was observed that the mass of the head and trunk segment of scoliotic girls was similar to that of able-bodied girls of similar age, height and weight. However its relative mass to that of the whole body was higher compared to able-bodied girls due to a smaller whole body mass. The horizontal position of the head and trunk segment's COM of scoliotic girls was more to the right and posterior compared to the able-bodied girls but their COM's height was similar. The AP COM offset was larger in scoliotic girls with the whole body COM anterior to that of the head and trunk segment. This could be explained by a greater pelvic forward tilt and a greater backward trunk inclination in scoliotic girls compared to able-bodied subjects. It appears that the COM position of the head and trunk is related to the trunk inclination in non-treated scoliotic girls. It is suggested to use the mean ratios obtained in this study to estimate the mass (i.e. 0.5538) and COM (i.e. 0.6438) of the head and trunk in scoliotic girls to overcome the drawbacks of current anthropometric methods, if direct measurements cannot be taken.

\section{Acknowledgement}

This work was funded in part by the Natural Sciences and Engineering Research Council of Canada (NSERC).

\section{Conflict of interest}

The author confirms that there is no conflict of interest in relation to this work.

\section{Funding}

This work was funded in part by the Natural Sciences and Engineering Research Council of Canada.

\section{Ethical approval}

Ethical approval was issued by Saint-Justine Hospital (affiliated with the University of Montreal), Montreal, Canada.

\section{References}

[1] Clauser CE, McConville JT, Young JW. Weight, volume, and center of mass of segments of the human body. AMRL technical report 69-70. OH: Wright Air Development Center, Wright Patterson Air Force Base; 1969.

[2] Dempster WT. Space requirements for the seated operator. WADC technical report 55-159. OH: Wright Air Development Center, Wright Patterson Air Force Base; 1955

[3] Jensen RK. Body segment mass, radius and radius of gyration proportions of children. J Biomech 1986;19:359-68.

[4] Reid JD, Jensen RK. Human body segment inertia parameters: a survey and status report. Exerc Sport Sci Rev 1990;18:225-41.

[5] Pearsall DJ, Reid JG, Ross R. Inertial properties of the human trunk of males determined from magnetic resonance imaging. Ann Biomed Eng 1994;22:692-706

[6] Allard P, Chavet P, Barbier F, Gatto L, Labelle H, Sadeghi H. Effect of body morphology on standing balance in adolescent idiopathic scoliosis. Am J Phys Med Rehabil 2004:83:689-97.

[7] Durkin JL, Dowling JJ. Analysis of body segment parameter differences between four human populations and the estimation errors of four popular mathematical models. J Biomech Eng 2003:125:515-22.

[8] Zatsiorsky VM, Seluyanov V. The mass and inertia characteristics of the main segments of the human body. In: Matsui H, Kobayashi K, editors. Biomechanics VIII-B. Champaign, IL: Human Kinetics Publishers Inc.; 1983. p. 1152-9.

[9] Damavandi M, Farahpour N, Allard P. Determination of body segment masses and centers of mass using a force plate method in individuals of different morphology. Med Eng Phys 2009;31:1187-94.

[10] Engsberg JR, Lenke LG, Hollander KW, Uhrich ML, Commean PK, Lee JR, et al Methods to locate center of gravity in scoliosis. Spine 2003;23:E483-9.

[11] de Leva P. Adjustments to Zatsiorsky-Seluyanov's segment inertial parameters. J Biomech 1996;29:1223-30.

[12] Hanavan EP. A mathematical model of the human body. AMRL technical report 64-102. OH: Aerospace Medical Research Laboratory, Wright Patterson Air Force Base; 1964.

[13] Jensen RK. Estimation of the biomechanical properties of three body types using a photogrammetric method. J Biomech 1978;11:349-58.

[14] Goldberg CJ, Kaliszer M, Moore DP, Fogarty EE, Dowling FE. Surface topography, Cobb angles, and cosmetic change in scoliosis. Spine 2001;26:E55-63.

[15] Jaremko JL, Poncet P, Ronsky J, Harder J, Dansereau J, Labelle H, et al. Indices of torso asymmetry related to spinal deformity in scoliosis. Clin Biomech 2002;17:559-68 
[16] Jackson RP, McManus AC. Radiographic analysis of sagittal plane alignment and balance in standing volunteers and patients with low back pain matched for age, sex and size: a prospective controlled clinical study. Spine 1994:19:1611-8.

[17] Mahaudens P, Detrembleur C, Mousny M, Banse X. Gait in adolescent idiopathic scoliosis: energy cost analysis. Eur Spine J 2009;18:1160-8.

[18] Gagnon M, Sicard C, Drouin G. Evaluation of loads on the lumbar spine with motion analysis techniques and a static planar model. In: Winter DA, Norman RW, Wells RP, Hayes KC, Patla EA, editors. Biomechanics IX-B. Champaign, IL: Human Kinetics; 1985. p. 44-9.

[19] Heiss DG, Pagnacco G. Effect of center of pressure and trunk center of mass optimization methods on the analysis of whole body lifting mechanics. Clin Biomech 2002;17:106-15.

[20] Weinstein SL. Adolescent idiopathic scoliosis: prevalence and natural history. In: Weinstein SL, editor. The pediatric spine: principle and practice, vol. 1. New York, NY: Raven Press; 1994. p. 463-78.

[21] Bunnel WP. The natural history of idiopathic scoliosis before skeletal maturity. Spine 1986;11:773-6.

[22] Murray MP, Seireg A, Scholz RC. Center of gravity, center of pressure and supportive forces during human activities. J Appl Physiol 1967;23:831-8.

[23] Wessberg P, Danielson BI, Willén J. Comparison of Cobb angles in idiopathic scoliosis on standing radiographs and supine axially loaded MRI. Spine 2006;31(26):3039-44.

[24] Preece SJ, Willan P, Nester CJ, Graham-Smith P, Herrington L, Bowker P. Variation in pelvic morphology may prevent the identification of anterior pelvic tilt. J Man Manip Ther 2008;16(2):113-7.

[25] Larivière C, Gagnon D. The L5/S1 joint moment sensitivity to measurement errors in dynamic 3D multisegment lifting models. Hum Mov Sci 1999; $18: 573-87$

[26] Abboud RJ, Rowley DI, Newton RW. Lower limb muscle dysfunction may contribute to foot ulceration in diabetic patients. Clin Biomech 2000;15:37-45.

[27] Putti AB, Arnold GP, Cochrane LA, Abboud RJ. Normal pressure values and repeatability of the Emed ST4 system. Gait Posture 2008;27(3):501-5.
[28] Dickstein R, Nissan M, Pillar T, Scheer D. Foot-ground pressure pattern of stand ing hemiplegic patients major characteristics and patterns of improvement. Phys Ther 1984;64:19-23.

[29] Fegoun ABE, Schwab F, Gamez L, Champain N, Skalli W, Farcy JP. Center of gravity and radiographic posture analysis: a preliminary review of adult volunteers and adult patients affected by scoliosis. Spine 2005;30:1535-40.

[30] Mac-Thiong JM, Labelle H, Charlesbois M, Huot MP, de Guise JA. Sagittal plane analysis of the spine and pelvis in adolescent idiopathic scoliosis according to the coronal curve type. Spine 2003;13:1404-9.

[31] Glassman SD, Berven S, Bridwell K, Horton W, Dimar JR. Correlation of radiographic parameters and clinical symptoms in adult scoliosis. Spine 2005;30:682-8

[32] Ramirez N, Johnston CE, Browne RH. The prevalence of back pain in children who have idiopathic scoliosis. J Bone Joint Surg Am 1997;79:364-8.

[33] Jackson RP, Peterson MD, McManus AC, Hales C. Compensatory spinopelvic balance over the hip axis and better reliability in measuring lordosis to the pelvic radius on standing lateral radiographs of adult volunteers and patients. Spine 1998;23:1750-67.

[34] Nault ML, Allard P, Hinse S, Le Blanc R, Caron O, Labelle H. Relationships between standing stability and body posture parameters in adolescent idiopathic scoliosis. Spine 2002;27:1911-7.

[35] Gum JL, Asher MA, Burton DC, Lai SM, Lambert LM. Transverse plane pelvic rotation in adolescent idiopathic scoliosis: primary or compensatory. Eur Spine J 2007;16:1579-86.

[36] Mahaudens P, Banse X, Mousny M, Detrembleur C. Gait in adolescent idiopathic scoliosis: kinematics and electromyographic analysis. Eur Spine J 2009; 18:512-21.

[37] Gillet C, Duboy J, Barbier F, Armand S, Jeddi R, Lepoutre FX, et al. Contribution of the accelerated body masses to able-bodied gait. Am J Phys Med Rehabil 2003;82:101-9.

[38] Dalleau G, Allard M, Beaulieu M, Rivard CH, Allard P. Free moment contribution to quiet standing in able-bodied and scoliotic girls. Eur Spine J 2007;16: 1593-9. 